Internat. J. Math. \& Math. Sci.

Vol. 23, No. 5 (2000) 319-334

S0161171200001745

(C) Hindawi Publishing Corp.

\title{
SIMILARITY ANALYSIS FOR NATURAL CONVECTION FROM A VERTICAL PLATE WITH DISTRIBUTED WALL CONCENTRATION
}

\author{
I. MULOLANI and M. RAHMAN
}

(Received 10 July 1998)

\begin{abstract}
Steady laminar natural convection flow over a semi-infinite vertical plate is examined in this paper. It is assumed that the concentration of a species along the plate follows some algebraic law with respect to chemical reaction. Similarity solutions may then be obtained for different orders of reaction. The fundamental parameters of this problem are the Schmidt number, Sc, and reaction order, $n$. Numerical results, based on the fourth order Runge-Kutta method, for Schmidt number ranging from 0.0 to 100.0 and reaction order from 0.0 to 1.5 are presented. When chemical reaction occurs, diffusion and velocity domains are seen to expand out from the plate. For large values of $n$, one may expect a smaller diffusion layer which, at fixed Schmidt number, is associated with increased velocity and reduced convection-layer.
\end{abstract}

Keywords and phrases. Natural convection, reaction, similarity, distributed, concentration.

2000 Mathematics Subject Classification. Primary 76R10.

1. Introduction. Natural convection flow exists in a variety of situations including porous media supported by surfaces. Such flows have application in a broad spectrum of engineering systems including geothermal reservoirs, building thermal insulation, direct-contact heat exchangers, solar heating systems, packed-bed catalytic reactors, nuclear waste disposal systems and enhanced recovery of petroleum resources (cf. [7]).

In many industrial applications, such as in cooling of electronic equipment, natural convection heat transfer is of high importance. The trend of miniaturization of electronic solid state devices has introduced problems of heat dissipation from the devices themselves, Wang [27]. Among the many possibilities for removing heat from an electronic device that involves conduction and/or convection (natural or forced) heat transfer, cooling with natural convection is considered as a cost effective and attractive technique because it is a convenient and inexpensive mode of heat transfer. Heat removal by this technique may be sufficient to keep weakly-heated electronic chips below a critical value set by reliability considerations. Efficient cooling cannot be achieved without understanding the heat transfer from each specific component and determining the flow and thermal fields (see Incropera [16]).

In many natural and technological processes, temperature and concentration differences occur simultaneously. Such processes occur in cleaning operations, drying, crystal growth, solar ponds and photosynthesis. A clear understanding of the nature of interaction between thermal and concentration buoyancies is necessary to control these processes (see Angirasa [3]). 
A problem of steady-state natural convection induced by chemical diffusion from a vertical plate has been reported by Levich [20]. The plate, at zero concentration of a chemical species $A$, but containing some catalytic substances, is placed in a fluid solution of $A$ at concentration $c_{0}>0$. When the plate comes in contact with the solution, a heterogeneous chemical reaction takes place on the plate. Changes in concentration imply density gradients which in the presence of a gravitational field, induce natural convection flow near the plate.

Recently, a numerical study of combined heat and mass transfer by natural convection adjacent to vertical surfaces situated in fluid-saturated porous media has been reported by Angirasa et al. [3]. Special attention was given to opposing buoyancy effects of the same order and unequal thermal and species diffusion coefficients.

Many principal past studies concerning natural convection flows over a semi-infinite vertical plate immersed in an ambient fluid where the flow is induced by both heat and chemical diffusion mechanisms have been found in the literature $[5,8,12,18]$. An interesting extension of this problem is the study of effects caused by the inclusion of general chemical reaction of order $n$. In particular, this study is concerned mainly with the steady-state behaviour of the same binary system composed of a semi-infinite plate and ambient fluid, each initially at different concentrations of a given species, but between which a homogeneous, irreversible, isothermal chemical reaction of order $n$ is assumed to occur. Similar studies have been pursued in the literature (see Meadley and Rahman [21], Gebhart and Pera [13]). This study serves to highlight and thus gain more insight into the effects of chemical diffusion and reaction on natural convection flow.

If a solid plate is immersed and held vertically within a fluid medium at rest, it may be possible to induce natural convection in the ambient fluid when the material species are such that chemical diffusion takes place between plate and fluid giving rise to a change in buoyancy forces. Despite the fact that the basic principles of heat and mass transfer are theoretically similar, the resulting flow pattern for each system may be quite distinct. A combination of the two mechanisms is also possible. Whatever the case, ultimate steady-state conditions may be established and maintained using external controls, Gebhart and Pera [13].

We present a theoretical study of laminar natural convection caused by chemical diffusion and reaction from a vertical plate surface. The focus of the study is on the essential nature of the flow and diffusion which occurs in the thin convection boundary-layer adjacent to the plate surface. For this, it is convenient to consider the idealised system composed of a semi-infinite plate set in a fluid of infinite extent. Such a model has been commonly used in previous work [11, 12, 18, 21] and permits the application of classical boundary-layer analysis in the mathematical formulation. In the analysis, steady two-dimensional laminar viscous flow over the semi-infinite vertical plate is examined. The constituents of the plate and the ambient fluid in which it is immersed undergo a homogeneous, isothermal, irreversible chemical reaction of $n$th order, Gebhart et al. [11].

2. Mathematical formulation. The principle of conservation of mass (fluid), conservation of momentum (fluid motion), and conservation of mass (species) by diffusion give the basic equations of natural convection flow. These equations can be written in 
the general form $[11,13]$

$$
\begin{gathered}
\frac{\partial \rho}{\partial t}+\vec{\nabla} \cdot(\rho \vec{V})=0, \\
\rho\left[\frac{\partial \vec{V}}{\partial t}+(\vec{V} \cdot \vec{\nabla}) \vec{V}\right]=\rho \vec{g}-\vec{\nabla} P+\mu \nabla^{2} \vec{V}+\frac{\mu}{3} \vec{\nabla}(\vec{\nabla} \cdot \vec{V}), \\
\frac{\partial C}{\partial t}+(\vec{V} \cdot \vec{\nabla}) C=\vec{\nabla} \cdot(D \vec{\nabla} C)+\dot{C}^{\prime \prime \prime} .
\end{gathered}
$$

Employing the Boussinesq approximation along with an order analysis, the equations for natural convection can be simplified even further to the form:

$$
\begin{gathered}
\frac{\partial U}{\partial X}+\frac{\partial V}{\partial Y}=0 \\
U \frac{\partial U}{\partial X}+V \frac{\partial U}{\partial Y}=g \beta^{*}\left(C-C_{\infty}\right)+v \frac{\partial^{2} U}{\partial Y^{2}}, \\
U \frac{\partial C}{\partial X}+V \frac{\partial C}{\partial Y}=-k\left(C-C_{\infty}\right)^{n}+D \frac{\partial^{2} C}{\partial Y^{2}} .
\end{gathered}
$$

The relevant boundary conditions are:

$$
\begin{aligned}
& \text { at } Y=0, \quad U=V=0, \\
& \text { at } Y \longrightarrow \infty, \quad U=V=0, C \longrightarrow 0 .
\end{aligned}
$$

Further, at the plate surface $Y=0$,

$$
C=C_{0}(X)
$$

Here, $C_{0}(X)$ is a given function of $X$ representing the concentration distribution along the plate. In this problem, $C_{0}(X)$ is considered to be arbitrary for the purpose of formulating the mathematical model. However, the nature of the function in reality may be subject to severe limitations which arise from the chemical kinetics involved in setting up steady-state conditions for a given species and ambient fluid (see Aris [4]). Introducing the stream function $\psi(X, Y)$ defined by

$$
U=\frac{\partial \psi}{\partial Y}, \quad V=-\frac{\partial \psi}{\partial X},
$$

equations (2.2) reduce to

$$
\begin{gathered}
\psi_{Y} \psi_{Y X}-\psi_{X} \psi_{Y Y}=v \psi_{Y Y Y}+g \beta^{*} C, \\
\psi_{Y} C_{X}-\psi_{X} C_{Y}=D C_{Y Y}-k C^{n}
\end{gathered}
$$

with boundary conditions

$$
Y=0:\left\{\begin{array}{l}
\psi_{Y}=\psi_{X}=0, \\
C=C_{0}(X),
\end{array} \quad Y \rightarrow \infty:\left\{\begin{array}{l}
\psi_{Y}=\psi_{X}=0, \\
C=0 .
\end{array}\right.\right.
$$

Now, we investigate the possibility of similarity solutions for this set of equations (see $[2,14])$. 
3. Basic equations for various reaction rates. Consider the following one-parameter transformation group

$$
\bar{X}=a^{m} X, \quad \bar{Y}=a^{l} Y, \quad \bar{\psi}=a^{p} \psi, \quad \bar{C}=a^{q} C,
$$

where " $a$ " is a parameter and the exponents $m, l, p$, and $q$ are constants. We can show that the governing equations can be transformed into a set of ordinary differential equations when the concentration at the surface of the plate follows an algebraic law. This is in comparison to the perturbation-type similarity solutions that arise in the case of uniform concentration along the plate.

CASE I. GENERAL $n$ TH ORDER CHEMICAL REACTION. Consider the transformations

$$
\begin{aligned}
& \eta(X, Y)=Y b(x), \quad \psi(X, Y)=v a(X) f(\eta), \\
& c(X, Y)=\frac{C-C_{\infty}}{C_{0}-C_{\infty}}, \quad e(X)=C_{0}-C_{\infty},
\end{aligned}
$$

and introduce them into (2.6) to obtain the differential equations.

$$
\begin{gathered}
f^{\prime \prime \prime}+\frac{g \beta^{*} e}{a b^{3} v^{2}} c+\frac{a_{x}}{b} f^{\prime \prime} f-\left(\frac{a b_{x}}{b^{2}}+\frac{a_{x}}{b}\right) f^{\prime 2}=0, \\
\frac{c^{\prime \prime}}{\mathrm{Sc}}+\frac{a_{x}}{b} f c^{\prime}-\frac{a e_{x}}{b e} f^{\prime} c-\frac{k e^{n-1}}{v b^{2}} c^{n}=0
\end{gathered}
$$

provided the following conditions hold:

$$
\begin{gathered}
\frac{a_{x}}{b}=C_{1}, \quad \frac{a b_{x}}{b^{2}}=C_{2}, \quad \frac{a e_{x}}{b e}=C_{3}, \\
\frac{g \beta^{*} e}{a b^{3} v^{2}}=C_{4}, \quad \frac{k e^{n-1}}{v b^{2}}=C_{5},
\end{gathered}
$$

where $C_{1}, C_{2}, C_{3}, C_{4}$, and $C_{5}$ are arbitrary constants. From these conditions, we need to determine expressions for $a(X), b(X)$, and $e(X)$ following the analysis of Gebhart et al. [11]. Taking the expressions in turn and integrating each one leads to the following result (see Gebhart et al. [11]):

$$
a(X)=\left(\frac{D\left(C_{1}-C_{2}\right)}{C_{1}}\right)^{C_{1} /\left(C_{1}-C_{2}\right)} X^{C_{1} /\left(C_{1}-C_{2}\right)} .
$$

It is then possible to derive the following expressions:

$$
e(X)=N X^{1 /(3-2 n)}, \quad c_{5}=p=k N^{n-1} \sqrt{\frac{6-4 n}{g \beta^{*} N}} .
$$

The differential equations for the $n$th order chemical reaction are given by

$$
\begin{gathered}
f^{\prime \prime \prime}+(5-3 n) f f^{\prime \prime}+c-(4-2 n) f^{\prime 2}=0, \\
\frac{c^{\prime \prime}}{\mathrm{Sc}}+(5-3 n) f c^{\prime}-2 f^{\prime} c-p c^{n}=0
\end{gathered}
$$

provided $n \neq 1,3 / 2,5 / 3$. We note that the order of reaction need not necessarily be an integer. The pyrolysis of acetaldehyde $(n=3 / 2)$, and the formation of phosgene 
from carbon monoxide and chlorine $(n=5 / 2)$ are a few of the frequently encountered fractional reaction orders. the decomposition of nitrogen pentoxide and the radioactive disintegration of unstable nuclei are examples of first order reactions while the gas phase thermal decomposition of nitrogen dioxide and hydrogen iodide are both examples of second order reactions. Third order reactions are extremely rare in engineering practice, an example being nitric oxide with chlorine and oxygen. The similarity transformations for these equations are given by:

$$
\begin{aligned}
\eta(X, Y) & =\frac{Y}{X}\left(\frac{\mathrm{Gr}_{x}}{6-4 n}\right)^{1 / 4}, \\
\psi(X, Y) & =v(6-4 n)\left(\frac{\mathrm{Gr}_{x}}{6-4 n}\right)^{1 / 4} f(\eta), \\
c(X, Y) & =\frac{C-C_{\infty}}{C_{0}-C_{\infty}} \\
e(X) & =C_{0}-C_{\infty}=N X^{1 /(3-2 n)} .
\end{aligned}
$$

CASE II. ZEROTH ORDER CHEMiCAL REACTION. From the $n$th order general case, we can derive the zeroth order chemical reaction differential equations. The differential equations become

$$
\begin{aligned}
& f^{\prime \prime \prime}+5 f f^{\prime \prime}+c-4 f^{2}=0, \\
& \frac{c^{\prime \prime}}{\mathrm{Sc}}+5 f c^{\prime}-2 f^{\prime} c-q=0,
\end{aligned}
$$

where

$$
q=k \sqrt{\frac{6}{g \beta^{*} N^{3}}} .
$$

The similarity transformations for this set of equations are given by:

$$
\begin{aligned}
\eta(X, Y) & =\frac{Y}{X}\left(\frac{\mathrm{Gr}_{x}}{6}\right)^{1 / 4}, \\
\psi(X, Y) & =6 v\left(\frac{\mathrm{Gr}_{x}}{6}\right)^{1 / 4} f(\eta), \\
c(X, Y) & =\frac{C-C_{\infty}}{C_{0}-C_{\infty}}, \\
e(X) & =N X^{1 / 3} .
\end{aligned}
$$

CASE III. Chemical ReAction of ORDer $n=1 / 2$. Again, from the general $n$th order case, the differential equations for a chemical reaction of order $n=1 / 2$ may be derived. They are

$$
\begin{gathered}
f^{\prime \prime \prime}+\frac{7}{2} f f^{\prime \prime}+c-3 f^{\prime 2}=0, \\
\frac{c^{\prime \prime}}{\mathrm{Sc}}+\frac{7}{2} f c^{\prime}-2 f^{\prime} c-R c^{1 / 2}=0,
\end{gathered}
$$

where

$$
R=\frac{2 k}{N \sqrt{g \beta^{*}}}
$$


and the transformations are given by:

$$
\begin{aligned}
\eta(X, Y) & =\frac{Y}{X}\left(\frac{\mathrm{Gr}_{x}}{4}\right)^{1 / 4} \\
\psi(X, Y) & =4 v\left(\frac{\mathrm{Gr}_{x}}{4}\right)^{1 / 4} f(\eta), \\
c(X, Y) & =\frac{C-C_{\infty}}{C_{0}-C_{\infty}} \\
e(X) & =N X
\end{aligned}
$$

CASE IV. Chemical ReAction OF ORDER $n=3 / 5$. From the general $n$th order case, the $n=3 / 5$ order chemical reaction may be derived. The differential equations for this case are

$$
\begin{aligned}
& f^{\prime \prime \prime}+\frac{16}{5} f f^{\prime \prime}+c-\frac{14}{5} f^{\prime 2}=0 \\
& \frac{c^{\prime \prime}}{\mathrm{Sc}}+\frac{16}{5} f c^{\prime}-2 f^{\prime} c-P c^{3 / 5}=0 .
\end{aligned}
$$

The similarity transformations are:

$$
\begin{aligned}
\eta(X, Y) & =\frac{Y}{X}\left(\frac{5 \mathrm{Gr}_{x}}{18}\right)^{1 / 4}, \\
\psi(X, Y) & =\frac{18 v}{5}\left(\frac{5 \mathrm{Gr}_{x}}{18}\right)^{1 / 4} f(\eta), \\
c(X, Y) & =\frac{C-C_{\infty}}{C_{0}-C_{\infty}} \\
e(X) & =N X^{5 / 9}
\end{aligned}
$$

CASE V. CHEMiCAL REACTION OF ORDER $n=6 / 5$. Again from the $n$th order chemical reaction, the case $n=6 / 5$ may be derived. The differential equations for this are

$$
\begin{aligned}
& f^{\prime \prime \prime}+\frac{7}{5} f f^{\prime \prime}+c-\frac{8}{5} f^{2}=0, \\
& \frac{c^{\prime \prime}}{\mathrm{Sc}}+\frac{7}{5} f c^{\prime}-2 f^{\prime} c-\mathrm{Sc}^{6 / 5}=0 .
\end{aligned}
$$

The similarity transformations are given by the following:

$$
\begin{aligned}
\eta(X, Y) & =\frac{Y}{X}\left(\frac{5 \mathrm{Gr}_{x}}{6}\right)^{1 / 4} \\
\psi(X, Y) & =\frac{6 v}{5}\left(\frac{5 \mathrm{Gr}_{x}}{6}\right)^{1 / 4} f(\eta) \\
c(X, Y) & =\frac{C-C_{\infty}}{C_{0}-C_{\infty}} \\
e(X) & =N X^{5 / 3}
\end{aligned}
$$

CASE VI. Chemical ReAction of ORder $n=3 / 2$. When the reaction order is $n=3 / 2$, we must derive the differential equations from the original equations (3.3). 
An analysis similar to that in Case I is repeated to obtain the following differential equations:

$$
\begin{gathered}
f^{\prime \prime \prime}+c+C_{1}\left(f f^{\prime \prime}-2 f^{2}\right)=0, \\
\frac{c^{\prime \prime}}{\mathrm{Sc}}+C_{1}\left(f c^{\prime}-4 f^{\prime} c\right)-r c^{3 / 2}=0,
\end{gathered}
$$

where

$$
r=\frac{k N^{1 / 2}}{\nu\left(g \beta^{*} N / \nu^{2}\right)^{2 / 3}} .
$$

The similarity transformations are

$$
\begin{aligned}
\eta(X, Y) & =Y\left(\frac{g \beta^{*} N}{v^{2}}\right)^{1 / 3} e^{\left(g \beta^{*} N / v^{2}\right)^{1 / 3} C_{1} X}, \\
\psi(x, Y) & =v e^{\left(g \beta^{*} N / v^{2}\right)^{1 / 3} C_{1} X} f(\eta), \\
e(X) & =N e^{4\left(g \beta^{*} N / v^{2}\right)^{1 / 3} C_{1} X} .
\end{aligned}
$$

To aid in the graphical analysis, these cases have been arbitrarily chosen, i.e., $n=$ $0,1 / 2,3 / 2,3 / 5,6 / 5,5 / 3$. These similarity transformations for the cases chosen are unique to this paper, whereas the method of obtaining the transforms is not (see Gebhart et al. [11]). The boundary conditions for these 6 cases are given by

$$
\eta=0:\left\{\begin{array}{l}
f(0)=0, \\
f^{\prime}(0)=0, \\
c(0)=1,
\end{array} \quad \eta \rightarrow \infty:\left\{\begin{array}{l}
f^{\prime}(\infty)=0 \\
c(\infty)=0 .
\end{array}\right.\right.
$$

4. Numerical solution method. These approximations have been integrated numerically for various Schmidt numbers and reaction orders using a Runge-Kutta integration scheme to correct for assumed starting values of the initial conditions at the surface. The general forms of the equations to be treated are:

$$
\begin{gathered}
f^{\prime \prime \prime}(\eta)=F\left(f^{\prime \prime}, f^{\prime}, g^{\prime}, f, g, \eta\right), \\
g^{\prime \prime}(\eta)=G\left(f^{\prime \prime}, f^{\prime}, g^{\prime}, f, g, \eta\right)
\end{gathered}
$$

with the initial and asymptotic boundary conditions (3.22). With the two asymptotic boundary conditions, it was necessary to assume starting values for the two additional conditions that were required. Let

$$
A=f^{\prime \prime}(0), \quad B=g^{\prime}(0)
$$

to fulfill the requirement that

$$
\begin{gathered}
\lim _{\eta \rightarrow \infty} f^{\prime}(A, B, \eta)=f_{\infty}^{\prime}(A, B)=0, \\
\lim _{\eta \rightarrow \infty} g(A, B, \eta)=g_{\infty}(A, B)=0 .
\end{gathered}
$$


If it is assumed that $A_{1}$ and $B_{1}$ are trial values of $A$ and $B$ such that

$$
A=A_{1}+h, \quad B=B_{1}+k,
$$

where $h$ and $k$ are small, thus by (4.3) we have

$$
\begin{aligned}
& f_{\infty}^{\prime}\left(A_{1}+h, B_{1}+k\right)=0, \\
& g_{\infty}\left(A_{1}+h, B_{1}+k\right)=0 .
\end{aligned}
$$

In addition, to satisfying the asymptotic boundary conditions, it was assumed that the gradients of (4.5) were zero at infinity. This leads to

$$
\begin{aligned}
& f_{\infty}^{\prime \prime}\left(A_{1}+h, B_{1}+k\right)=0, \\
& g_{\infty}^{\prime}\left(A_{1}+h, B_{1}+k\right)=0 .
\end{aligned}
$$

Taylor's expansions for small $h$ and $k$ were then applied to (4.5) and (4.6). In matrix form, the problem may be expressed as:

$$
\left(\begin{array}{ll}
\frac{\partial f_{\infty}^{\prime}}{\partial A} & \frac{\partial f_{\infty}^{\prime}}{\partial B} \\
\frac{\partial g_{\infty}}{\partial A} & \frac{\partial g_{\infty}}{\partial B} \\
\frac{\partial f_{\infty}^{\prime \prime}}{\partial A} & \frac{\partial f_{\infty}^{\prime \prime}}{\partial B} \\
\frac{\partial g_{\infty}^{\prime}}{\partial A} & \frac{\partial g_{\infty}^{\prime}}{\partial B}
\end{array}\right)\left(\begin{array}{l}
h \\
k
\end{array}\right)=-\left(\begin{array}{l}
f_{\infty}^{\prime}(A, B) \\
g_{\infty}(A, B) \\
f_{\infty}^{\prime \prime}(A, B) \\
g_{\infty}^{\prime}(A, B)
\end{array}\right) .
$$

The application of the least squares method yields the least square error

$$
E=f_{\infty}^{\prime 2}+g_{\infty}^{2}+{f^{\prime \prime}}_{\infty}^{2}+g_{\infty}^{\prime}{ }^{2} .
$$

The partial derivatives appearing in the solutions of $h$ and $k$ can be obtained by integrating the perturbed differential equations with their appropriate initial conditions. From (4.1) we obtain the perturbed differential equations for the $A$-derivatives with the initial conditions as shown in the following:

$$
\begin{aligned}
\frac{\partial f^{\prime \prime \prime}}{\partial A} & =\frac{\partial F}{\partial f^{\prime \prime}} \frac{\partial f^{\prime \prime}}{\partial A}+\frac{\partial F}{\partial f^{\prime}} \frac{\partial f^{\prime}}{\partial A}+\frac{\partial F}{\partial g^{\prime}} \frac{\partial g^{\prime}}{\partial A}+\frac{\partial F}{\partial f} \frac{\partial f}{\partial A}+\frac{\partial F}{\partial g} \frac{\partial g}{\partial A}, \\
\frac{\partial g^{\prime \prime}}{\partial A} & =\frac{\partial G}{\partial f^{\prime \prime}} \frac{\partial f^{\prime \prime}}{\partial A}+\frac{\partial G}{\partial f^{\prime}} \frac{\partial f^{\prime}}{\partial A}+\frac{\partial G}{\partial g^{\prime}} \frac{\partial g^{\prime}}{\partial A}+\frac{\partial G}{\partial f} \frac{\partial f}{\partial A}+\frac{\partial G}{\partial g} \frac{\partial g}{\partial A}, \\
\eta & =0:\left\{\begin{array}{l}
\frac{\partial f}{\partial A}=\frac{\partial f^{\prime}}{\partial A}=\frac{\partial g^{\prime}}{\partial A}=\frac{\partial g}{\partial A}=0, \\
\frac{\partial f^{\prime \prime}}{\partial A}=1 .
\end{array}\right.
\end{aligned}
$$

Perturbed differential equations for the $B$-derivatives with appropriate initial conditions were obtained from (4.1). To correct the trial values of $A_{1}$ and $B_{1}$, the original equations (4.1), (4.2) with the perturbed equations for $A$ and $B$ with their appropriate initial conditions were integrated simultaneously up to a certain suitable point. At this point, the trial values of $A_{1}$ and $B_{1}$ were corrected to refine the solution. After two or three iterations at the same point where the least-square error appeared to be steady, the integration range was extended and the process repeated up to the extended point. This iterative process was continued until the desired solution accuracy was obtained. 
5. Discussion of results. From the results, it is observed that decreasing the Schmidt number increases the velocity level and reduces the concentration diffusion region. In Figure 5.1, the effect of the order of reaction on concentration is shown as

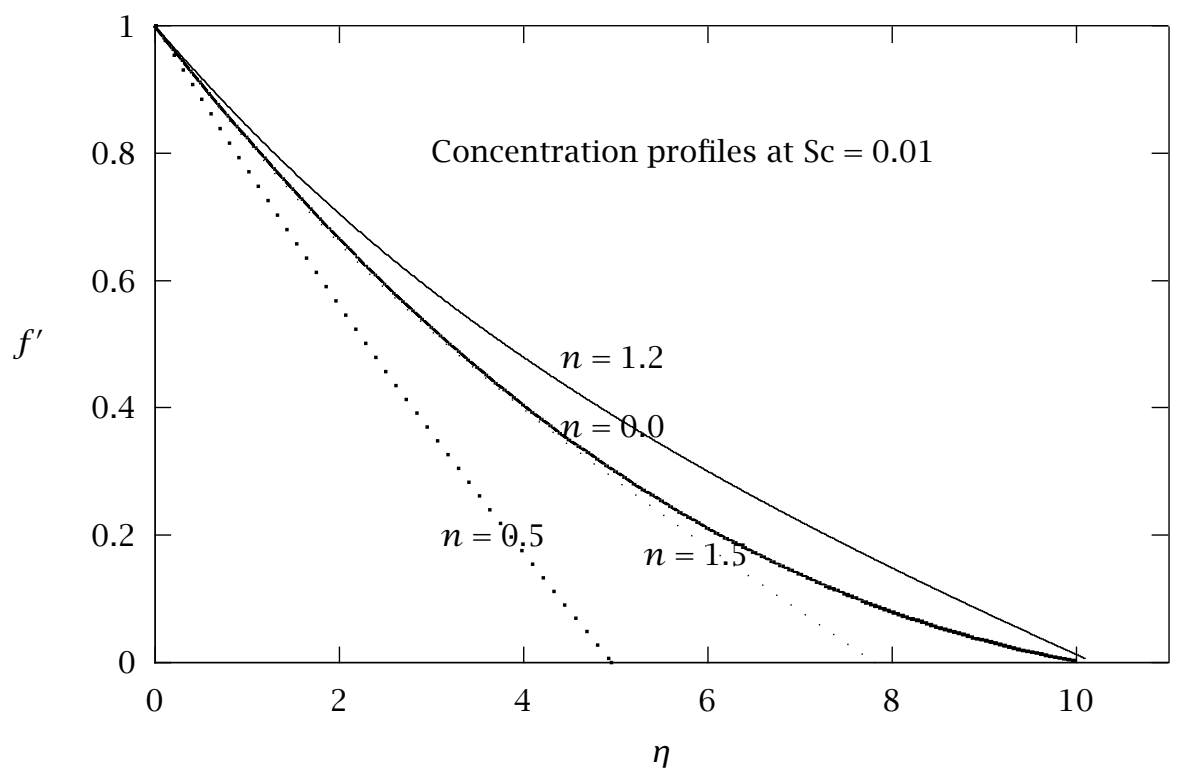

FIGURE 5.1. Effect of rate of reaction on concentration.

$n$ varies from $n=0,0.5,1.2$ at fixed Sc $=0.01$. Within the range $(0,2)$, an increase of $n$ (which effectively increases the sensitivity of species depletion with change in concentration) tends to decrease the concentration diffusion region to about $50 \%$ of its value before increasing slightly. Overall, the concentration gradient increases. We can expect a smaller less distinct diffusion layer for larger orders of $n$. This agrees keeping with the analysis made by Meadley and Rahman [21]. Figure 5.2 shows the effect of the rate of reaction on the velocity for orders $n=0,0.5,1.2,1.5$ at Sc $=0.01$. Here, the maximum velocity decreases slightly before rising to a value above its initial level. On the other hand, the extent of the convection layer is reduced to a little over $50 \%$ of its original size which is to be expected since the concentration of species on the plate increases as the length of the plate is traversed in the vertical direction. Flow reversal is also expected as $n$ increases (see Figures 5.2 and 5.9) and the Schmidt number decreases. Examination of the equations for each of the Cases I-VII, reveals that as Schimdt number increases to a considerable amount $(1000 \leq \mathrm{Sc})$, the second order differential term's effect $\left(c^{\prime \prime}\right)$ is neglected. To take its effect into consideration, a singular perturbation expansion of the concentration equation in each case would be needed.

6. Conclusions. This study has mainly been concerned with obtaining similarity solutions of natural convection flows induced by a semi-infinite vertical plate with distributed concentration along the plate wall. The basis of this theoretical work has been classical boundary-layer analysis. 


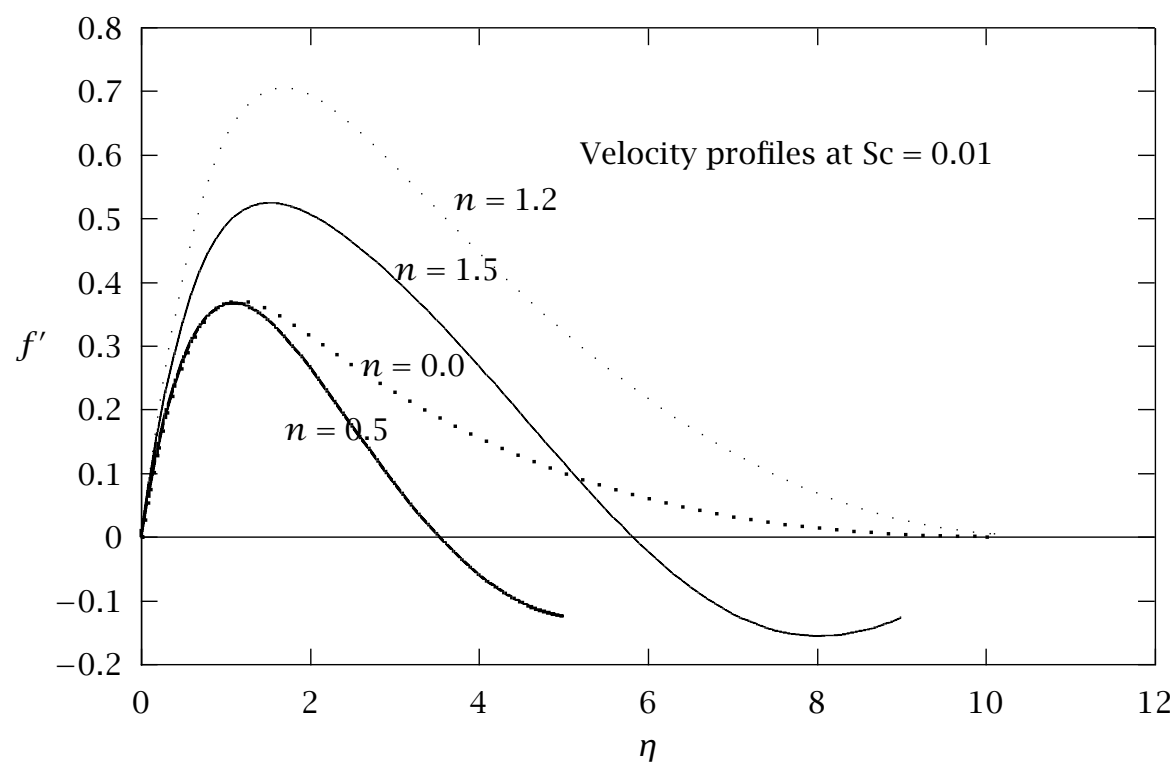

FIGURE 5.2. Effect of rate of reaction on velocity.

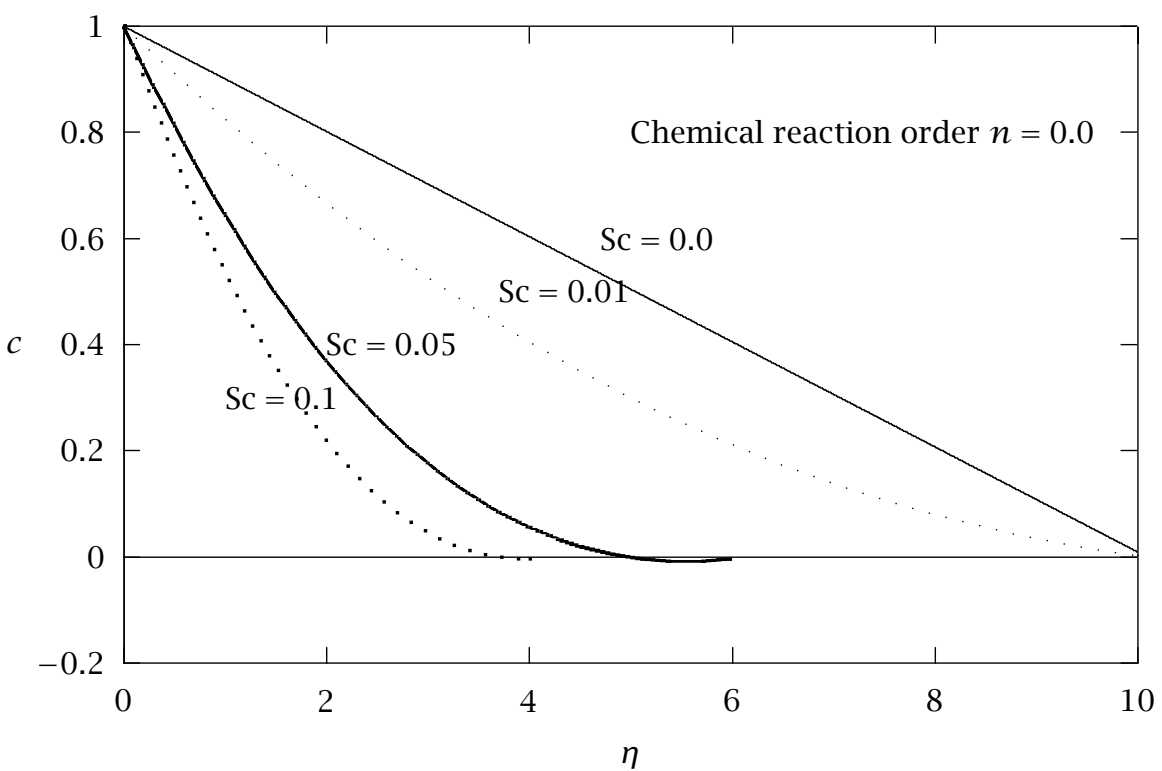

FIGURE 5.3. Zeroth order concentration profiles.

Steady-state natural convection flow over a semi-infinite vertical plate has been studied for the case in which the plate is of a given concentration in a chemical species and convection arises as a result of chemical reaction and diffusion within the ambient fluid. Similarity solutions have been obtained for various orders of chemical 


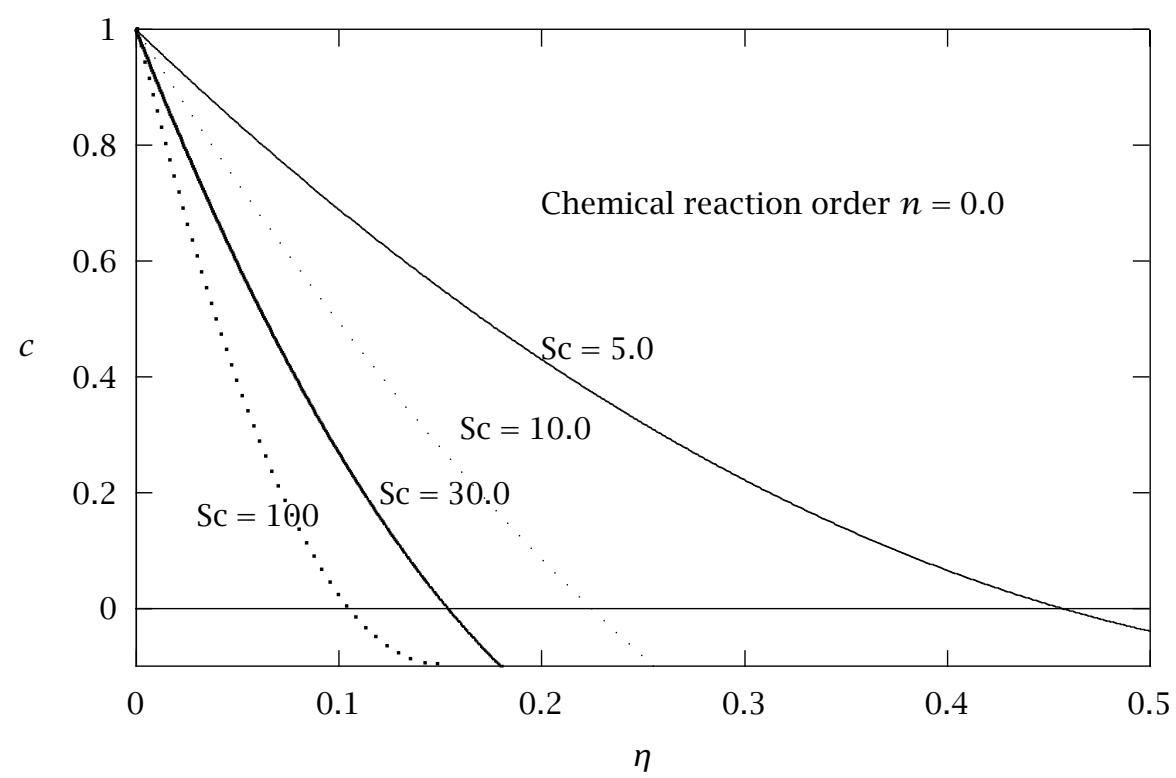

FIGURE 5.4. Zeroth order concentration profiles.

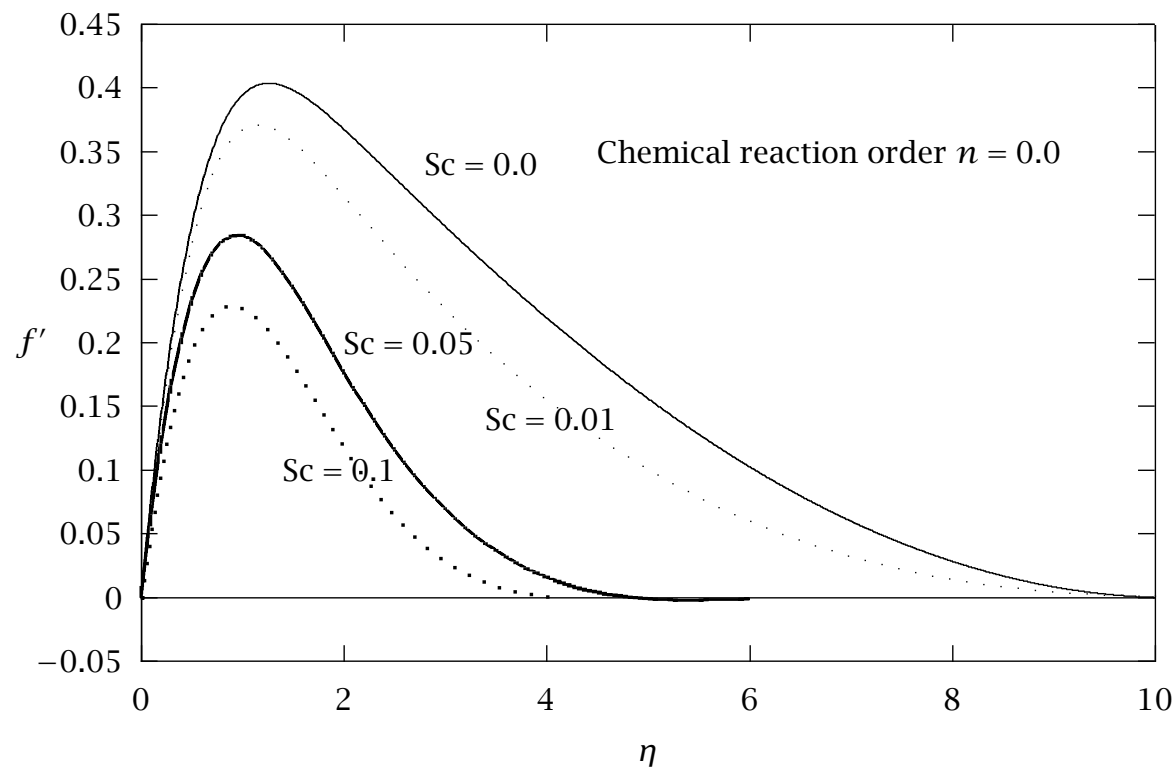

FIGURE 5.5. Zeroth order velocity profiles.

reaction when the concentration of species along the plate follows some algebraic law (power law).

It has been found that increasing the order of reaction $n$, increases the concentration gradient. For large values of $n$, a smaller diffusion layer is expected. Also, this increase 


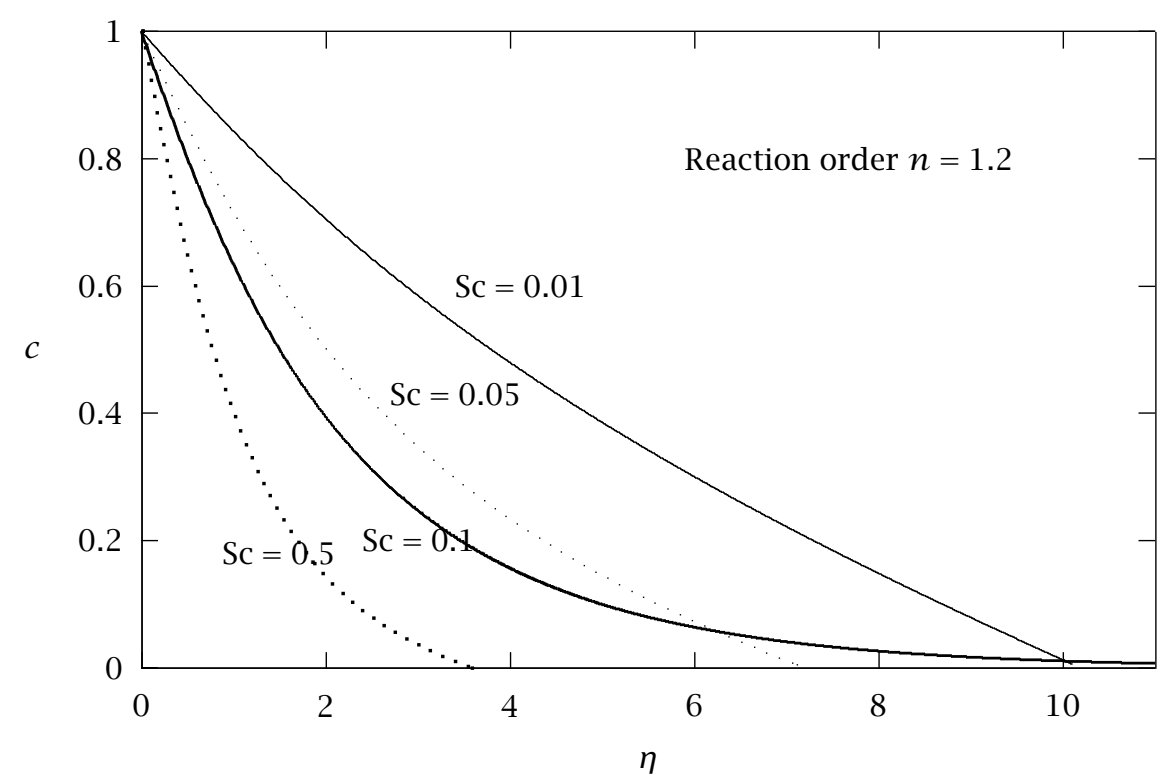

FIGURE 5.6. Concentration profiles for $n=1.2$.

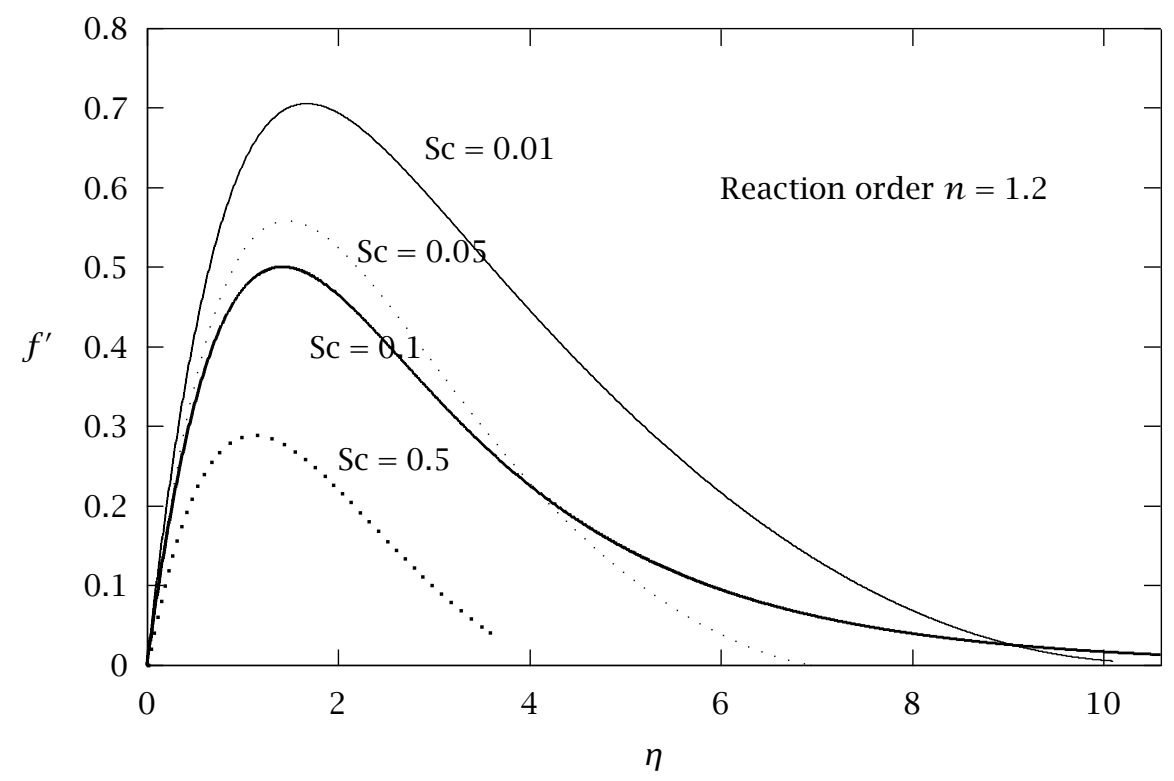

FIGURE 5.7. Velocity profiles for $n=1.2$.

in reaction order, $n$, at fixed Schmidt number is associated with increased velocity and reduced convection-layer.

Further study of this problem could investigate the effects of higher Schimdt numbers on the reaction rate and mass diffusion process. Such an investigation would necessarily need to incorporate a singular perturbation expansion in the equation for 


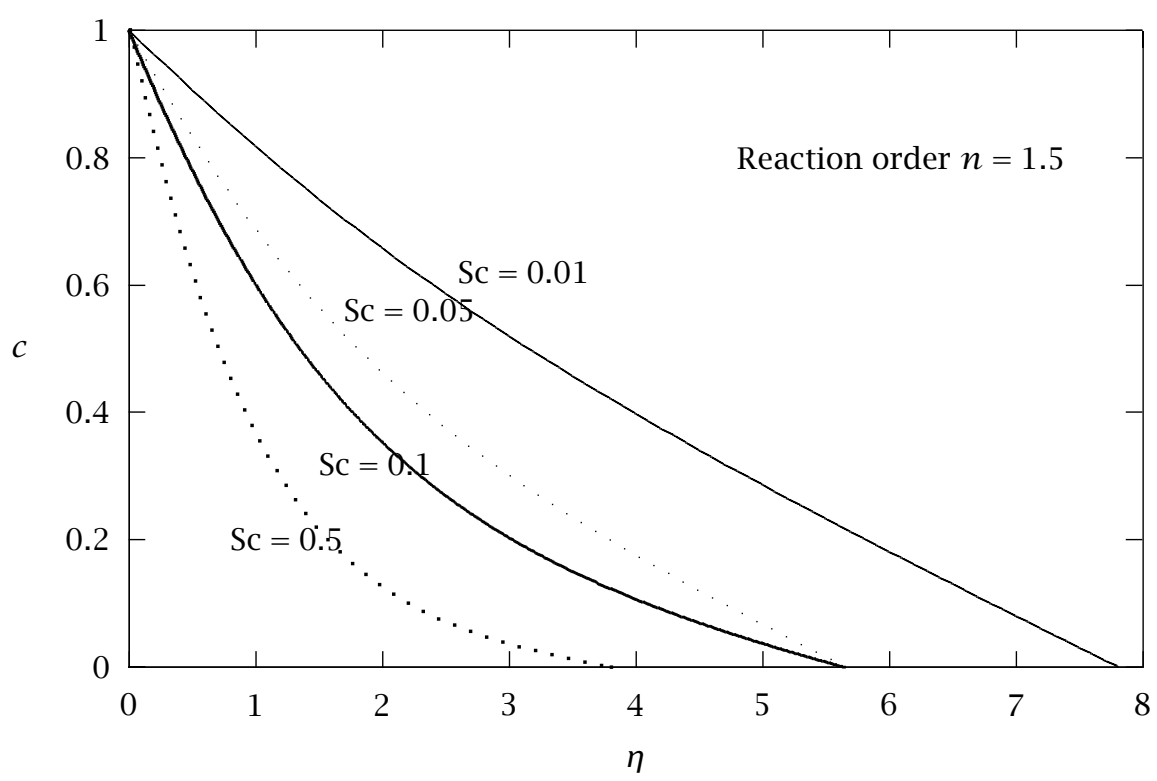

FIGURE 5.8. Concentration profiles for $n=1.5$.

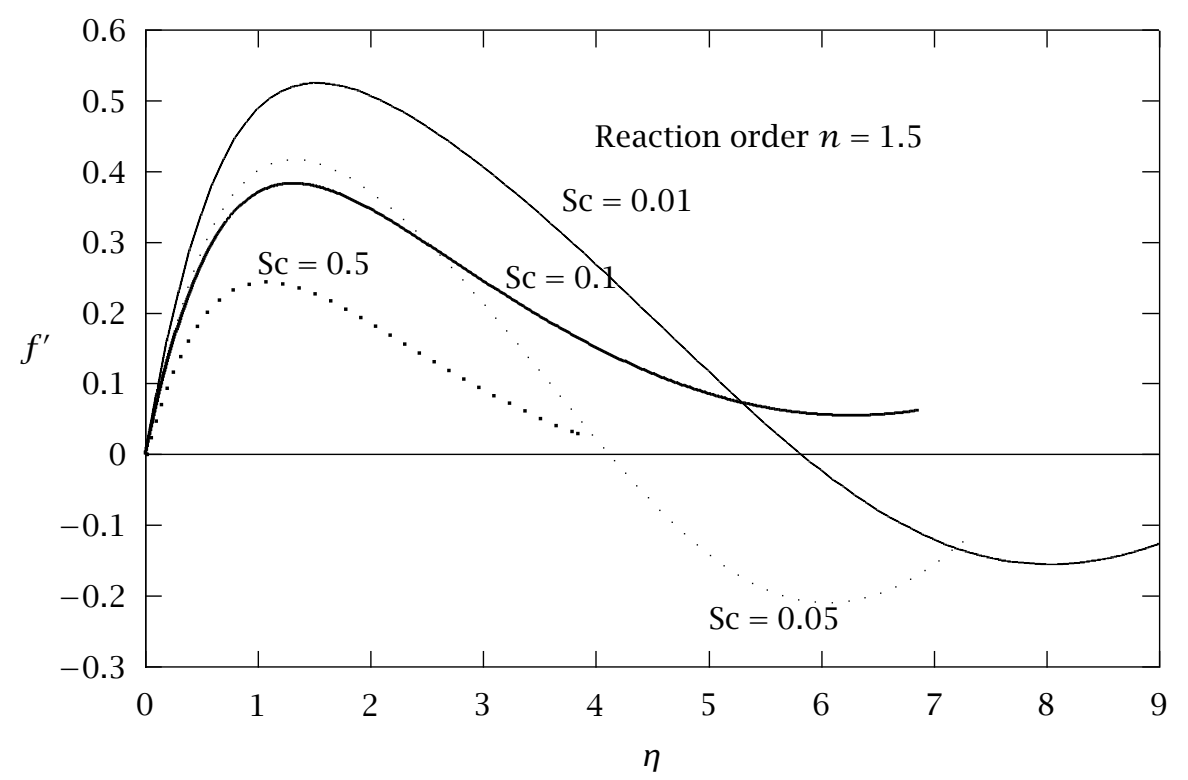

FIGURE 5.9. Velocity profiles for $n=1.5$.

the concentration. A matched asymptotic expansion method is needed to carry out this investigation.

ACKNowledgement. The authors are grateful to the Natural Sciences and Engineering Research Council of Canada for the financial support leading to this paper. 
NOMENCLATURE. In the following list of symbols, characteristic dimensions are given in terms of mass $(M)$, length $(L)$, temperature $(T)$, and velocity $\left(U_{\mathcal{C}}\right)$.

\section{LATIN CHARACTERS:}

$C=$ dimensional species concentration

$C_{0}=$ species concentration at vertical plate

$C_{\infty}=$ species concentration in the ambient fluid

$c=C-C_{0} / C_{0}-C_{\infty}=$ nondimensional species concentration

$C_{\rho}=$ specific heat of fluid at constant pressure

$\dot{C}^{\prime \prime \prime}=$ chemical reaction rate

$D=$ chemical molecular diffusivity

$f=$ nondimensional stream function

$g=$ acceleration due to gravity

$\mathrm{Gr}_{x}=$ Grashof number

$J^{\prime \prime}=$ mass flux (moles)

$k=$ homogeneous chemical rate constant

$K=$ heat conduction coefficient

$n=$ order of reaction

$\mathrm{Nu}=$ Nusselt number

$\operatorname{Pr}=$ Prandtl number

$P=$ fluid pressure

$\mathrm{Re}=$ Reynolds number

Sc $=$ Schmidt number

$T=$ absolute temperature of fluid

$U=$ velocity in $x$-direction

$U_{c}=$ characteristic velocity

$V=$ velocity component in $y$-direction

$\vec{V}=$ velocity vector

$X=$ vertical distance along the plate surface

$Y=$ horizontal distance from the plate surface.

\section{GREEK CHARACTERS:}

$\alpha=K / \rho C_{\rho}=$ thermal diffusivity

$\beta=$ thermal coefficient of volumetric expansion

$\beta^{*}=$ volumetric coefficient of expansion with concentration

$\mu=$ dynamic viscosity of fluid

$v=\mu / \rho=$ kinematic viscosity of fluid

$\rho=$ fluid density

$\psi=$ stream function

$\eta=$ similarity variable

$\epsilon=$ dimensionless reaction number

$\vec{\nabla}=$ the "del" operator

$\nabla^{2}=\partial^{2} / \partial X^{2}+\partial^{2} / \partial Y^{2}+\partial^{2} / \partial Z^{2}$. 


\section{REFERENCES}

[1] H. I. Abu-Mulaweh, B. F. Armaly, and T. S. Chen, Laminar natural convection flow over a vertical backward-facing step, Trans. ASME Ser. C J. Heat Transfer 117 (1995), 895-901.

[2] W. F. Ames, Nonlinear Partial Differential Equations in Engineering, Academic Press, New York, 1965. MR 35\#1235. Zbl 176.39701.

[3] D. Angirasa, G. P. Peterson, and I. Pop, Combined heat and mass transfer by natural convection with opposing buoyancy effects in a fluid saturated porous medium, Int. J. Heat Mass Transfer 40 (1997), no. 12, 2755-2773. Zbl 925.76653.

[4] R. Aris, Introduction to the Analysis of Chemical Reactors, Prentice-Hall, Inc., New Jersey, 1965.

[5] A. Bejan and J. L. Lage, The Prandtl number effect on the transition in natural convection along a vertical surface, J. Heat Transfer 112 (1990), 787-790.

[6] R. A. Brown, Fluid Mechanics of the Atmosphere, Academic Press, Inc., San Diego, 1991.

[7] A. J. Chamkha, Solar radiation assisted natural convection in uniform porous medium supported by a vertical flat plat, J. Heat Transfer 119 (1997), 89-96.

[8] R. Cheesewright, Turbulent natural convection from a vertical plane surface, Trans. Am. Soc. Mech. Engrs. C 90 (1968), 1-8.

[9] T. Fujii, Comments in the paper"Convection naturelle turbulente sur une plaque verticle isotherme, transition, echange de chaleur et frottement parietal, lois de repartition de vitesse et de temperature", Int. J. Heat Mass Transfer 14 (1971), 1876-1878.

[10] B. Gebhart, Heat Transfer, McGraw-Hill Book Co., New York, 1961.

[11] B. Gebhart, Y. Jaluria, R. L. Mahajan, and B. Sammakia, Bouyancy-Induced Flows and Transport, Hemisphere Publishing Corporation, 1988. Zbl 699.76001.

[12] B. Gebhart and J. Mollendorf, Viscous dissipation in external natural convection flows, J. Fluid Mech. 38 (1969), 97-107. Zbl 179.57001.

[13] B. Gebhart and L. Pera, The nature of vertical natural convection flows resulting from the combined buoyancy effects of thermal and mass diffusion, Int. J. Heat Mass Transfer 14 (1971), 2025-2049. Zbl 223.76060.

[14] A. G. Hansen, Similarity Analyses of Boundary Value Problems in Engineering, PrenticeHall Inc., Englewood Cliffs, N.J., 1964. MR 31\#2853. Zbl 137.22603.

[15] R. A. W. M. Henkes and C. J. Hoogendoorn, Laminar natural convection boundary-layer flow along a heated vertical plate in a stratified environment, Int. J. Heat Mass Transfer 32 (1989), no. 1, 147-155. Zbl 662.76108.

[16] F. P. Incropera, Convection heat transfer in electronic equipment NC-cooling, Trans. ASME Ser. C J. Heat Transfer 110 (1988), 1097-1111.

[17] Y. Joshi and B. Gebhart, Vertical transient natural convection flows in cold water, Int. J. Heat Mass Transfer 27 (1984), no. 9, 1573-1582.

[18] A. K. Kulkarni, H. R. Jacobs, and J. J. Hwang, Similarity solution for natural convection flow over an isothermal vertical wall immersed in thermally stratified medium, Int. J. Heat Mass Transfer 30 (1987), 691-698.

[19] S. Lee and M. M. Yovanovich, Laminar natural convection from a vertical plate with a step change in wall temperature, J. Heat Transfer 113 (1991), 501-504.

[20] V. G. Levich, Physico-Chemical Hydrodynamics, Prentice-Hall Inc., Englewood Cliffs, N.J., 1962.

[21] C. K. Meadley and M. Rahman, Laminar natural convection caused by chemical diffusion and reaction from a vertical plane surface, Can. J. Chem. Eng. (1974), 552-557.

[22] J. H. Merkin, A note on the similarity solutions for free convection on a vertical plate, J. Engrg. Math. 19 (1985), no. 3, 189-201. MR 87d:76135. Zbl 577.76083.

[23] J. H. Merkin and T. Mahmood, On the free convection boundary layer on a vertical plate with prescribed surface heat flux, J. Engrg. Math. 24 (1990), no. 2, 95-107. MR 91b:76094. Zbl 708.76119. 
[24] I. Mulolani, Similarity analysis of natural convection flow due to chemical diffusion, Master's thesis, DalTech, 1997.

[25] M. S. Raju, X. Q. Liu, and C. K. Law, A formulation of combined forced and free convection past horizontal and vertical surfaces, Int. J. Heat Mass Transfer 27 (1984), no. 12, 2215-2224. Zbl 554.76066.

[26] J. Shu and G. Wilks, Mixed-convection laminar film condensation on a semi-infinite vertical plate, J. Fluid Mech. 300 (1995), 207-229. MR 96e:76120. Zbl 848.76091.

[27] H. Y. Wang, F. Penote, and J. B. Sauliner, Numerical study of buoyancy-induced flow along a vertical plate with discretely heated integrated circuit packages, Int. J. Heat Mass Transfer 40 (1997), no. 7, 1509-1520. Zbl 925.76411.

Mulolani ANd Rahman: Department of Applied Mathematics, Daltech, Dalhousie UniVERSITY, P.O. BOX 1000, HALIFAX NS, B3J 2X4, CANADA 


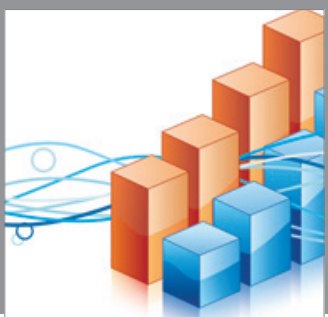

Advances in

Operations Research

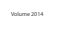

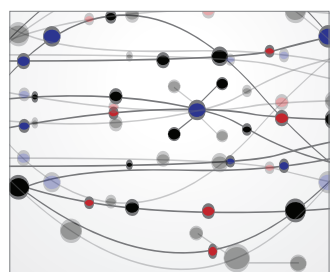

\section{The Scientific} World Journal
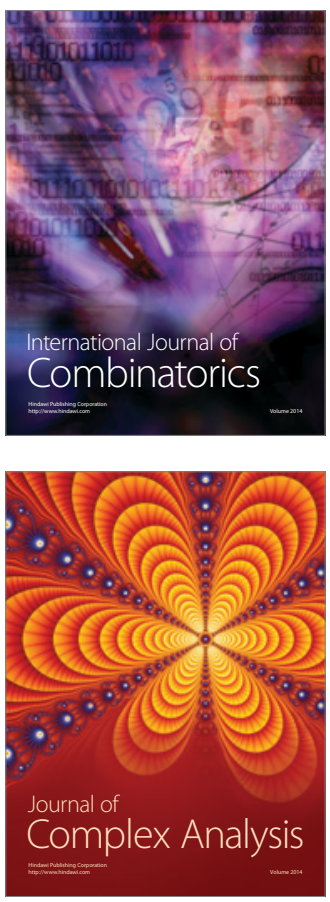

International Journal of

Mathematics and

Mathematical

Sciences
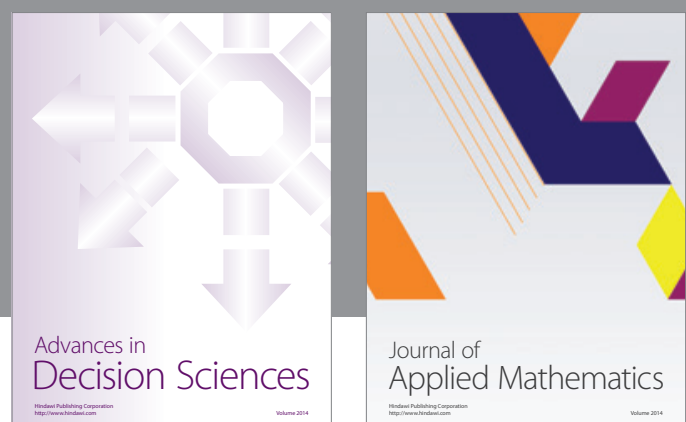

Journal of

Applied Mathematics
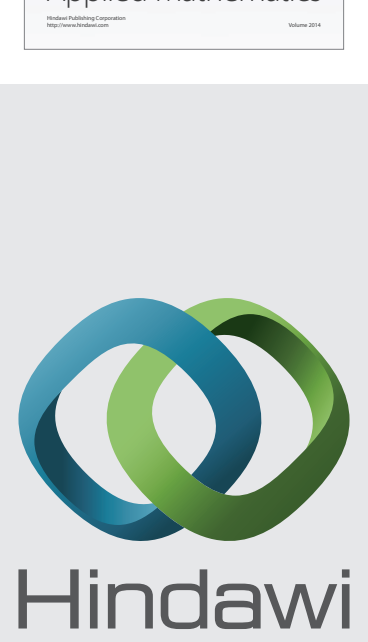

Submit your manuscripts at http://www.hindawi.com
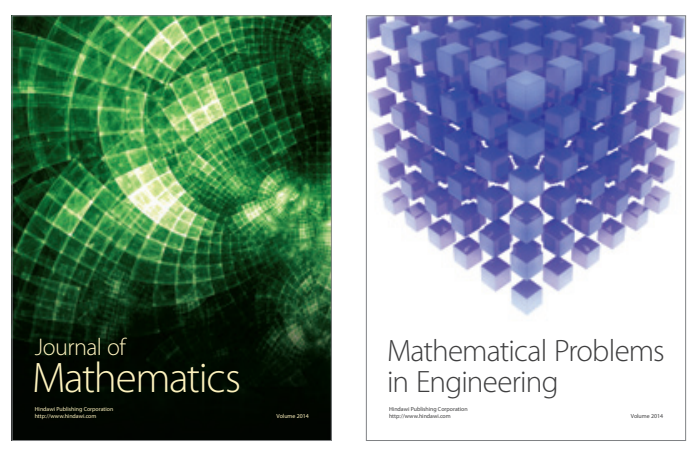

Mathematical Problems in Engineering
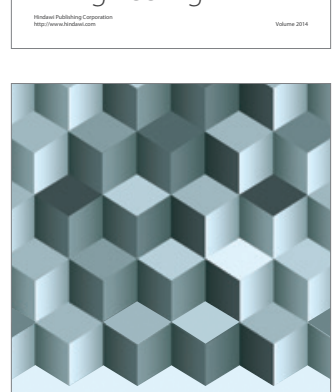

Journal of

Function Spaces
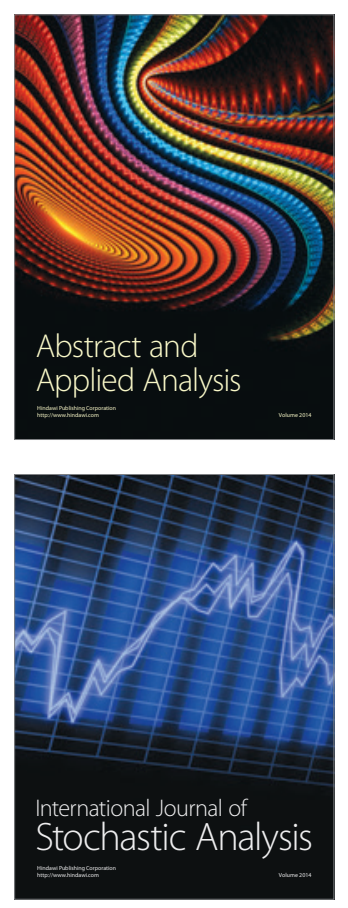

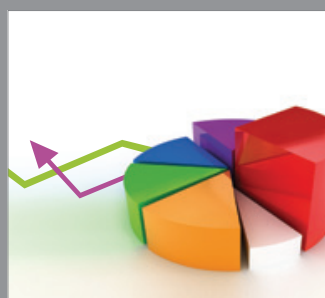

ournal of

Probability and Statistics

Promensencen
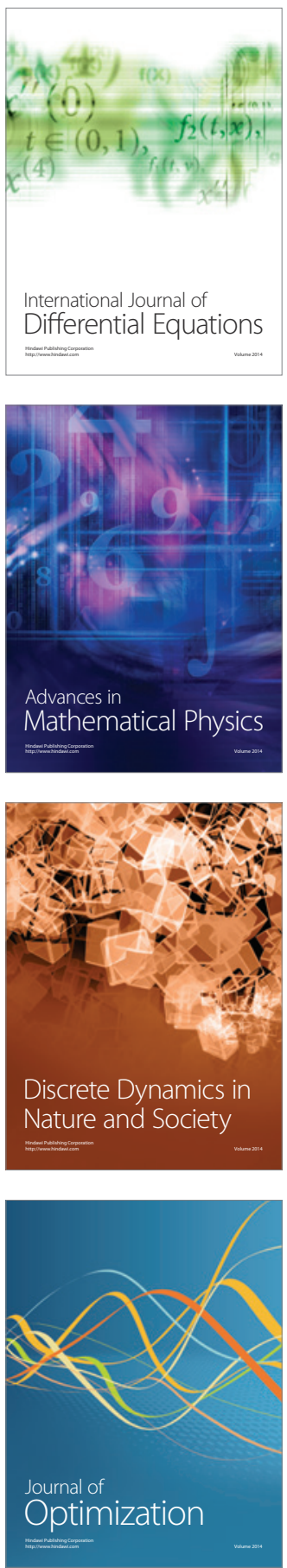